\title{
A Hypothesis of the Magnetostatic Turbulence and its Implications for Astrophysics
}

D.D. Ryutov, B.A. Remington

February 27, 2007

International Workshop "Collective Phenomena in Macroscopic Systems"

Como, Italy

December 4, 2006 through December 6, 2006 
This document was prepared as an account of work sponsored by an agency of the United States Government. Neither the United States Government nor the University of California nor any of their employees, makes any warranty, express or implied, or assumes any legal liability or responsibility for the accuracy, completeness, or usefulness of any information, apparatus, product, or process disclosed, or represents that its use would not infringe privately owned rights. Reference herein to any specific commercial product, process, or service by trade name, trademark, manufacturer, or otherwise, does not necessarily constitute or imply its endorsement, recommendation, or favoring by the United States Government or the University of California. The views and opinions of authors expressed herein do not necessarily state or reflect those of the United States Government or the University of California, and shall not be used for advertising or product endorsement purposes. 


\title{
A HYPOTHESIS OF THE MAGNETOSTATIC TURBULENCE AND ITS IMPLICATIONS FOR ASTROPHYSICS*
}

\author{
D.D. RYUTOV, B.A. REMINGTON \\ Lawrence Livermore National Laboratory, Livermore, CA 94551, USA
}

\begin{abstract}
Arguments are presented in favor of a possible existence of a random, force-free magnetic field. Ponderomotive forces in such a field are small, and the evolutionary time is much longer than Alfven crossing time over the vortex scale, whence the suggested term "magnetostatic." The presence of this long-lived random magnetic field provides stiffness with respect to large-scale compressional motions. On the other hand, such a field cannot be detected by techniques involving line-of-sight averaging. It may therefore be a source of stiffness for various astrophysical objects, ranging from plasmas in clusters of galaxies to the interiors of molecular clouds in HII regions, and remaining at the same time undetectable. Analysis of large-scale motions on the background of the magnetostatic turbulence is presented; it is concluded that these large-scale motions can be roughly described by a usual hydrodynamics for the matter with an isotropic pressure; the adiabatic index is $4 / 3$.
\end{abstract}

\section{Introduction}

Consider a low-pressure plasma, with a random, highly entangled magnetic field immersed into it. By a "low pressure" we mean a plasma where condition $p<<B^{2} / 8 \pi$ is satisfied, with $p$ being a plasma pressure, and $B$ being the magnetic field strength (we are using CGS system of units). If "set loose," such a system will very quickly come to the state of a violent turbulence, with characteristic velocities of order of the Alfven velocity (evaluated for the r.m.s. value of the magnetic field). Because of the assumed smallness of the plasma pressure, the turbulence will be compressible.

Compressible MHD turbulence has been extensively studied during the past decade (e.g., $[1,2])$, with the conclusion that the initial magnetic energy is dissipated very quickly, within approximately one turn-over time $l / \mathrm{v}_{\mathrm{A}}$, where $l$ is the characteristic vortex size and $v_{\mathrm{A}}$ is the Alfven velocity. The magnetic energy is converted into the thermal energy of the plasma via the shock formation and magnetic reconnection.

On the other hand, it has been hypothesized [3-5] that, instead of relaxing to the state of a low magnetic energy, in some cases the system may relax to an intermediate, long lived transitional state where the magnetic field strength will remain comparable to its initial value, but the structure of the field

\footnotetext{
" This work was performed under the auspices of the U.S. Department of Energy by University of California Lawrence Livermore National Laboratory under contract No. W-7405-Eng-48.
} 
would change to an essentially force-free field. In other words, the current will be approximately aligned with the magnetic field, so that $\left(\boldsymbol{B} \cdot \boldsymbol{\nabla} \times \boldsymbol{B}<<B^{2} / l\right.$, where $l$ is the characteristic vortex size). The magnetic field will remain tangled, randomly changing its direction, so that the averaging of the field over the scale much greater than $l$ will yield zero. Such a state, if it exists, can be called the state of "magnetostatic" turbulence, because the evolution time for it will be much larger than the inertial time $l / \mathrm{v}_{\mathrm{A}}$.

If magnetostatic turbulence indeed exists, it may have significant implications for interpretation of a wide variety of astrophysical phenomena, from the behavior of hot matter in cluster of galaxies [6], to the dynamics of molecular clouds in HII regions (e.g., [7,8]). The magnetostatic turbulence would provide a high "stiffness" to the matter in such objects, without allowing the observer to detect easily the existence of the magnetic field. In particular, the synchrotron radiation will be un-polarized. Also, the Faraday rotation (that is proportional to the line-of sight integral) will be absent because of the randomness of the magnetic field. So, the hypothesis of the magnetostatic turbulence may help in resolving some of the observational paradoxes.

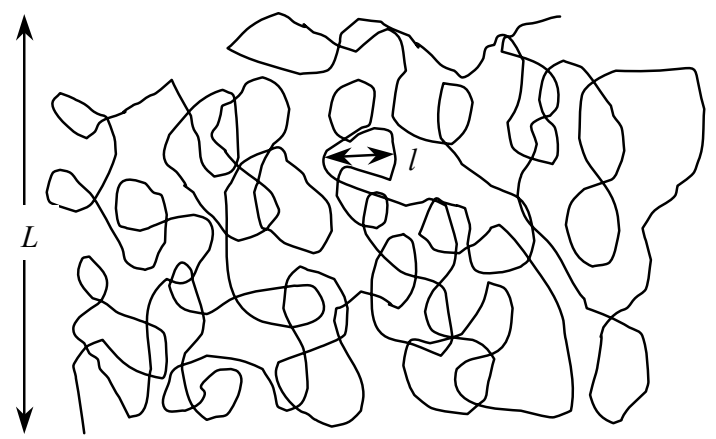

Figure 1. Field line of a random magnetic field. Self-intersections are caused by the projection effect. The correlation length $l$ is much less than the global scale $L$.

In this paper we consider purely MHD aspects of the magnetostatic turbulence. The issues of the applicability of MHD equations in various settings have been considered in Refs. [3, 9]. Although there exist some constraints, the magnetohydrodynamic description should be applicable in typical astrophysical settings, mainly due to very large spatial scales involved (the typical mean free path is extremely small compared to the scale length). The medium that we consider has a low electrical resistivity in the sense that resistive dissipation time is long compared to the dynamical time. 


\section{Existence of magnetostatic turbulence}

Here we present arguments that show that the state of a random, force-free field does indeed exist. Consider initial state where random (but not a force-free) field is present in some finite volume limited by perfectly conducting walls (Fig. 1).

Let this initial state evolve according to the following set of equations:

$$
\begin{aligned}
& \frac{\boldsymbol{j} \times \boldsymbol{B}}{c}-\alpha \mathbf{v}=0, \\
& \frac{\partial \boldsymbol{B}}{\partial t}=\nabla \times[\mathbf{v} \times \boldsymbol{B}], \\
& \nabla \times \boldsymbol{B}=\frac{4 \pi}{c} \boldsymbol{j} .
\end{aligned}
$$

This is a set of MHD equations for a medium experiencing a friction force characterized by the friction coefficient $\alpha$. The pressure is assumed to be negligibly small; the friction coefficient is large enough to make inertial forces negligible. Equations (1)-(3) play an auxiliary role in our analysis: we use them to show that the random, force-free magnetic field may indeed exist; we do not claim that they describe any real astrophysical system.

If we set this system loose, it starts moving. Its dynamics is subject to two exact relations that follow directly from the set (1)-(3):

$$
\frac{\partial}{\partial t}\left(\int \frac{B^{2}}{8 \pi} d V\right)=-\int \alpha \mathrm{v}^{2} d V,
$$

and

$$
\int \boldsymbol{A} \cdot \boldsymbol{B} d V=\left.\left[\int \boldsymbol{A} \cdot \boldsymbol{B} d V\right]\right|_{t=0}
$$

where $\boldsymbol{A}$ is the vector-potential, $\nabla \times \boldsymbol{A}=\boldsymbol{B}$. Equation (4) is the energy integral, whereas Eq. (5) describes helicity conservation (e.g., [10]).

Consider the energy integral. The right-hand side in Eq. (4) is negativedefinite. Therefore, the magnetic energy is a decreasing function of time. If we assume that $\mathbf{v}$ remains non-zero, we come to a contradiction: the magnetic energy would have to become negative at a large-enough time. Therefore, we conclude that, asymptotically, the set of equations (1)-(3) brings the system to a state where $\mathbf{v}=0$. This, in turn, by virtue of Eq. (1), means that, asymptotically, the system reaches a force-free state, in which $\boldsymbol{j} \times \boldsymbol{B}=0$. On the other hand, the magnetic field in this state is non-zero, because of the helicity conservation. Therefore, we conclude that the final state is a non-trivial state, with a finite value of the magnetic field. It is natural to assume that the characteristic correlation length $l$ of the force-free field remains of the same order as in the initial state. 
In order to ensure that the r.m.s. value of this field would be not much smaller then the r.m.s. of the initial field, the helicity integral must be largeenough. Specifically, if the r.m.s. value of the initial magnetic field was $B_{0}$, and the correlation length was $l_{0}$, then the initial value of the helicity integral should be of order

$$
\left.\left[\int \boldsymbol{A} \cdot \boldsymbol{B} d V\right]\right|_{t=0} \sim B_{0}^{2} l_{0}^{4}\left(L^{3} / l_{0}^{3}\right) \sim B_{0}^{2} l_{0} L^{3} .
$$

What is assumed here is that contributions to the overall helicity integral from "cells" of a scale $l_{0}, B_{0}^{2} l_{0}^{4}$, are of the same sign for all cells, so that the total helicity integral can be evaluated by multiplying this quantity by the number of "cells" in a macroscopic volume $L$, i.e., by $\left(L^{3} / l_{0}^{3}\right)$, yielding the estimate (6). If the contributions of various "cells" had randomly distributed signs, then the initial helicity would have been almost zero, thereby not imposing any meaningful constraint on the final amplitude of the magnetic field.

Although we have not proven that the field remains random, it is hard to imagine that initial random field would become regular. So, this discussion provides a support to our hypothesis that random force-free fields do exist. In the final state of the system described by the set (1)-(3) current sheets may be present on the background of an otherwise smooth current distribution (which has a characteristic scale $l$ ).

The approach based on the use of auxiliary Eqs. (1)-(3) may help in numerical assessment of the possible structure of the random, force free field: numerical simulation of the set (1)-(3) is much simpler than simulation of the set where Eq. (1) would be replaced by a full dynamic equation; in the latter case, the continuity equation, together with the equation of state, would be also required.

In the exactly force-free state, the condition of collinearity $\boldsymbol{j}$ and $\boldsymbol{B}$ should be satisfied, meaning that

$$
\nabla \times \boldsymbol{B}=\lambda \boldsymbol{B}
$$

where $\lambda$ is some scalar function of coordinates. The condition $\nabla \cdot \boldsymbol{B}=0$ leads to a constraint

$$
\boldsymbol{B} \cdot \nabla \lambda=0
$$

meaning that $\lambda$ must be constant along the field line. However, if one assumes that the magnetic field slightly deviates from the exactly force-free field (as is the case in any real system), then slow variation of $\lambda$, such that

$$
\frac{l}{\lambda} \mid \nabla \lambda k<1
$$

becomes permissible. 


\section{Possible mechanisms generating magnetostatic turbulence}

Based on the analysis of the previous section, the existence of magnetostatic turbulence seems quite plausible. However, one has still to consider scenarios that would lead to the formation of this state in real dynamical systems. One possibility is that the system is initially driven to a state of a strong, non-forcefree MHD turbulence, and then gradually evolves to the force-free state by dissipating initial energy via formation of shocks and dumping the excessive heat via radiation. This mechanism may, in particular, work in the case of molecular clouds in HII regions [4, 11]. In numerical simulations of such a scenario, one should make certain that, in the initial state, the helicity is large, approaching the value described by Eq. (6). This would make impossible a quick "unwinding" and rapid annihilation of the initial random magnetic field.

Another scenario would be associated with a gradual tangling of the initial seed magnetic field by slow random flows, that would slowly stretch and twist the magnetic field. All along this slow build-up process, relaxation events bringing the system to almost force-free state would occur. In this scenario, the system remains close to the force-free state in the course of a slow build-up process. As the $\boldsymbol{j} \times \boldsymbol{B}$ forces in this case will be much smaller than $B^{2} / 8 \pi l$, even a weak turbulent drive would allow for building up a strong (but almost forcefree!) magnetic field.

\section{Dissipation of the magnetostatic turbulence}

The Lundquist number $S \equiv l \mathrm{v}_{A} / D_{M}$ (where $\mathrm{v}_{\mathrm{A}}$ is the Alfven velocity, and $D_{M}$ is the magnetic diffusivity) is usually very large in astrophysical systems, mostly due to very large spatial scales involved. Note that we define $S$ for the scale of turbulent vortices $l$, not the global scale $L$, but $S$ is still quite large for all moreor-less realistic assumptions. Accordingly, the Ohmic dissipation time for the turbulent vortices, $l S / \mathrm{v}_{A}$, is very large compared to the Alfven transit time $l / \mathrm{v}_{A}$ and is typically too long to be of any interest. More relevant time-scale is set by reconnection processes.

In the Sweet-Parker model $[12,13]$ the reconnection time $\tau$ scales as

$$
\tau \sim l \sqrt{S} / \mathrm{v}_{A},
$$

whereas in the Petschek model [14] reconnection occurs much faster,

$$
\tau \sim\left(l / \mathrm{v}_{A}\right) \ln S .
$$

It is difficult to say which of the two limits would be applicable to the magnetostatic turbulence. If the condition (9) holds, the parameter $\lambda$ is almost constant over a large number of neighboring cells, meaning that locally this state is one of the Taylor states [10]. This would mean that reconnection process 
would be inhibited. So, it is probable that the magnetostatic turbulence would exist for the times much longer than Petschek reconnection time (11). But even if reconnection occurs on the time-scale (11), it is still relatively slow (because $S$ is large), and even a modest drive would sustain magnetostatic turbulence against dissipation processes.

\section{Large-scale motions on the background of the magnetostatic turbulence}

The term "force-free" that we have been using in the previous sections may be somewhat confusing when one considers large-scale motions. In fact, in order to prevent the medium carrying a random, force free magnetic field from expanding, one has to apply a confining force at its boundaries. If set loose, the medium would expand, as there will appear cross-field currents, first near the boundaries, and then, as the expansion continues, also in the inner regions. This is a consequence of a virial theorem (e.g., [15]). When the magnetostatic turbulence is uniform, there is no gradient of the average magnetic pressure, but as soon as some volumes get compressed (or stretched) the pressure gradient appears.

In this section we consider reaction of magnetostatic turbulence on the large-scale motions, e.g., compression of a volume containing a large number of cells. We present the corresponding analysis for the case of isotropic turbulence. Consider the normal component of the momentum flux through a surface perpendicular to axis $z$. The general expression for the $p_{z z}$ component of the flux is [16]:

$$
p_{z z}=\frac{B_{x}^{2}+B_{y}^{2}-B_{z}^{2}}{8 \pi} .
$$

We average this expression over the area covering many cells. In the isotropic case,

$$
\left\langle B_{x}^{2}\right\rangle=\left\langle B_{y}^{2}\right\rangle=\left\langle B_{z}^{2}\right\rangle=\left\langle B^{2}\right\rangle / 3
$$

In the isotropic case, the average (over many cells) stress tensor is isotropic, with the averaged pressure being $p=\left\langle p_{x x}\right\rangle=\left\langle p_{y y}\right\rangle=\left\langle p_{z z}\right\rangle$. Equations (12) and (13) then show that

$$
p=\frac{\left\langle B^{2}\right\rangle}{24 \pi} .
$$

On the other hand, the magnetic energy density is

$$
w_{M}=\frac{\left\langle B^{2}\right\rangle}{8 \pi} .
$$

Therefore, we conclude that the following relation holds:

$$
p=\frac{w_{M}}{3} \text {. }
$$


So, if the magnetostatic turbulence is the only source of the stiffness, its effect on the large-scale dynamics can be described as an effect of a polytropic gas [17] with the polytrope index

$$
\gamma=4 / 3 \text {. }
$$

This is an important conclusion, because it provides a tool for a quantitative description of large-scale compressional motion.

In addition to compressional forces, hear stresses may also appear if large-scale shear deformations are created in the medium with magnetostatic turbulence. We consider shear stress for the geometry of Fig. 2, where the plane 1 is displaced by the distance $\delta x$ in the $x$ direction with respect to plane 2 . The magnitude of the arising shear stress depends on the characteristic time of the motion. If deformation occurs on a time-scale short compared to the time required for the system to relax to a force-free state, then shear stress $\sigma_{x z}$ is

$$
\sigma_{x z}=-\xi W_{M} \frac{\partial \delta x}{\partial z},
$$

where $\xi$ is a numerical coefficient of order one. If, however, the deformation is slow, on the time-scale greater than $\tau$, then the relaxation reduces the stresses. This effect can be qualitatively described by a rheological equation

$$
\sigma_{x z}+\tau \frac{\partial \sigma_{x z}}{\partial t}=-\xi \tau W_{M} \frac{\partial}{\partial z}\left(\frac{\partial \delta x}{\partial t}\right)
$$

One sees that, in addition to compressional waves, the medium under consideration can be bearing also large-scale shear waves.



Figure 2. The shearing perturbation. Plane 1 is displaced by the distance $\delta x$ with respect to plane 2 in a conducting medium carrying magnetostatic turbulence. Thiss displacemet causes the appearance of the $x$ component of the restoring force.

\section{Discussion}

We have shown that there are good reasons to believe that magnetostatic turbulence does indeed exist. Its presence provides compressional stiffnes even 
to the medium where the gaseous pressure is negligible. Therefore, it serves as a surrogate gaseous pressure. On the other hand, the random magnetic field with a small-enough correlation length cannot be detected by the standard techniques which include the line-of sight integration. In particular, the synchrotron radiation of relativistic electrons would be un-polarized, the Faraday rotation will be absent, and polarization of light by dust grains would also be averaged out. \{At high-enough energies of relativistic electrons, the standard synchrotron radiation would be replaced by a "jitter" radiation [18], which would also be unpolirized.\} So, the presence of the magnetostatic turbulence with small-enough correlation length is hard to notice. Therefore, it may provide a natural solution to the problems of a high stiffness of a medium where the gaseous pressure is small and, at the same time, there is no indication on the presence of a regular magnetic field.

In this context it is also important to note that the life-time of magnetostatic turbulence will be significantly longer (at least by a factor of 1020) than one Alfvenic turn-over time $l / \mathrm{v}_{\mathrm{A}}$. Therefore, in the decay mode, magnetostatic turbulence will be a long-lived object (unlike its "standard" counterpart, for which the decay time is of order of $l / \mathrm{v}_{\mathrm{A}}$ ). In the driven mode, where the turbulence is supported by some external sources, the drive required for sustaining a certain level of the magnetic field will be small compared to the "standard" case.

Another facet of the magnetostatic turbulence is related to possible experimental simulations of various astrophysical phenomena with high-power lasers (see, e.g., [19]). It is usually difficult to introduce dynamically-significant magnetic fields in such experiments (see, e.g., [19]). However, in the case where one wants to imitate large-scale phenomena involving magnetostatic turbulence, one can do that without introducing any magnetic fields, just by selecting the matter with equation of state yielding the adiabatic constant of $4 / 3$.

With all this said, one should remember that possible properties of the magnetostatic turbulence have been discussed based largely on qualitative arguments. In the future, a number of issues will have to be studied in a more rigorous way. In particular, this relates to the evaluation of the decay time and possible role of singularities in the current distribution.

In the context of specific astrophysical systems, a more clear answer regarding the source of the magnetostatic turbulence is desirable. The processes responsible for establishing a correlation length $l$ have to be identified. Specific settings where the model may provide new insights include enhanced momentum transport generated by shear stresses, in particular, in the systems with sheared rotation. 


\section{References}

1. J.M. Stone, E.C. Ostriker, C.F. Gammie, Ap.J. 508, L99 (1998); E.C. Ostriker, J.M. Stone, C.F. Gammie, Ap.J. 546, 980 (2001).

2. M.M. MacLow et al.Phys. Rev. Lett. 80, 2754 (1998).

3. D.D. Ryutov, B.A. Remington, Plasma Phys. Contr. Fus. 44, B407 (2002).

4. D.D. Ryutov et al. AIP Conference Proceedings v. 703 (Eds.: Giuseppe Bertin, Daniela Farina, and Roberto Pozzoli), Melville, New York, 2004, pp. 415-424;

5. D.D. Ryutov et al. Astrophys. Space Sci. 298, 183-190 (2005).

6. A.C. Fabian. AIP Conference Proceedings v. 703, (Eds.: Giuseppe Bertin, Daniela Farina, and Roberto Pozzoli), Melville, New York, 2004, p.p. 337344.

7. M.W. Pound, Ap.J. 493, L113 (1998).

8. M.W. Pound, B. Reipurth, J. Bally, Astron. J. 125, 2108 (2003).

9. R.M. Kulsrud, W.P. Pierce, Ap.J, 156, 445 (1969).

10. J.B. Taylor, Phys. Rev. Lett. 33, 1139.

11. D.A. Neufield, S. Lepp, G.J. Melnick, Ap.J. Suppl. 100, 132 (1995).

12. P. A. Sweet, in Electromagnetic Phenomena in Cosmic Physics, edited by B. Lehnert (Cambridge University Press, London, 1958), p. 123.

13. E. N. Parker, Ap.J. Suppl. 8, 177 (1963).

14. E. Petschek, in AAS-NASA Symposium of the Physics of Solar Flares, NASA-SP 50, edited by W. N. Hess (National Aeronautics and Space Administration, Washington, DC, 1964), p. 425.

15. V.D. Shafranov, in Reviews of Plasma Physics, edited by M.A. Leontovich (Consultants Bureau, New York, 1965), vol. 2, p.112.

16. J.D. Jackson. Classical Electrodynamics (Chichester: Wiley), 1975.

17. L.D. Landau, E.M. Lifshitz, Fluid Mechanics, (New York, Pergamon), 1987. 18. M.V. Medvedev, Ap.J. 540, 704 (2000).

19. B.A. Remington, R.P. Drake, D.D. Ryutov, Rev. Mod. Phys., 78, 755, (2006). 